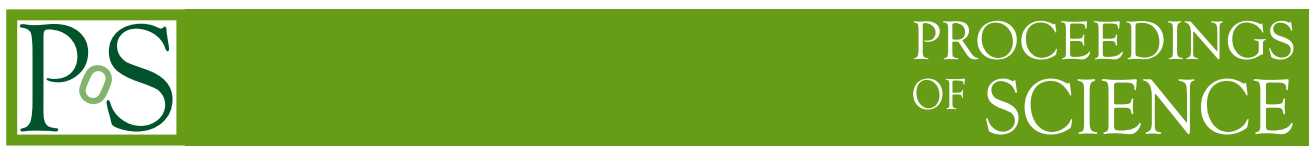

\title{
Late-Type Compact Objects
}

\author{
Elizabeth J. Jeffery* \\ James Madison University \\ 901 Carrier Dr., MSC 4502 \\ Harrisonburg, VA 22801, USA \\ E-mail: jeffe2ejejmu.edu
}

The final stages of stellar evolution for a low mass star are fairly unspectacular when compared to their high mass siblings. Once the outer layers of a star are shed, the hot exposed core that's left behind and begins to cool. As it does so, it exhibits several interesting (and useful!) character traits. In this paper I will give an overview of the discovery of white dwarf stars and what we now understand about their formation and place within the story of stellar evolution. The fate of 98\% of the stars in our Galaxy, white dwarfs continue to prove their usefulness and versatility as probes of understanding a number of interesting astrophysical problems. I will discuss a few of these applications, with particular emphasis on work done studying white dwarfs in star clusters.

Frank N. Bash Symposium New Horizons In Astronomy,

October 9-11, 2011

Austin Texas

*Speaker. 


\section{Introduction}

A white dwarf star, as we now know today, is the end point of a low mass star's life. Although only about the size of the Earth, their average mass is about $0.6 \mathrm{M}_{\odot}$, making them extremely dense. They are typically composed of mostly carbon and oxygen, with a thin layer of helium and maybe hydrogen surrounding the $\mathrm{C} / \mathrm{O}$ core. Nuclear fusion is no longer powering this type of star; the core is supported against further gravitational collapse by the electron degeneracy pressure.

While interesting in their own right, white dwarfs also serve astronomers in a variety of ways, as a probe to many different astrophysically interesting problems. In this paper I will discuss a variety of science questions that are and can be addressed with white dwarf stars. The range of their applications is a testament to their versatility as astrophysical probes.

This paper is arranged as follows: In Section 2 I briefly review the evolution of a low mass star to its ultimate fate as a white dwarf. In Section 3 I then discuss the different applications of white dwarfs, arranged roughly in order of temperature: interesting problems relating to hot, "warm," and cool white dwarfs. I particularly emphasize the use of WDs in star clusters, an area that has become more observationally feasible over the last 20 (or so) years with the use of large ground-based telescopes, as well as space-based telescopes, such as the Hubble Space Telescope. I summarize the discussion in Section 4.

\section{A White Dwarf's Place in Stellar Evolution}

The first white dwarf (WD) stars that were discovered were found as companions to brighter stars. They revealed themselves by the gravitational tugs they exerted on their companion stars. By measuring the periodic wobble of the brighter star, the smaller WD's mass can be measured. We now understand that WDs are the end point of a low mass star's evolution.

\subsection{The H-R Diagram and the Evolution of Low Mass Stars}

Ejnar Hertzsprung and Henry Norris Russell independently discovered relationships between a star's intrinsic brightness and its temperature. These patterns are most easily seen on what later became known as the Hertzsprung-Russell (H-R) Diagram, named in honor of its cofounders. The first H-R Diagram included a WD star (the companion star to 40 Eridani), although Russell dismissed it as a most likely an anomaly. (See Figure 1, taken from [26].) Of this "outlier" he said: "It is immediately conspicuous that one corner of the diagram is vacant (except for one star whose spectrum is very doubtful)." [25] More modern H-R Diagrams show that the lower left corner of the diagram, once thought to be vacant, is actually populated by the WDs.

All stars begin life when a cloud of gas and dust collapses to the point that temperatures and pressures in the core are hot and high enough to ignite hydrogen fusion. A star spends most of its life on the main sequence (MS), and will stay in this stage until it has exhausted its core hydrogen supply. When this occurs, the star's core (made now mostly of helium, the by- 


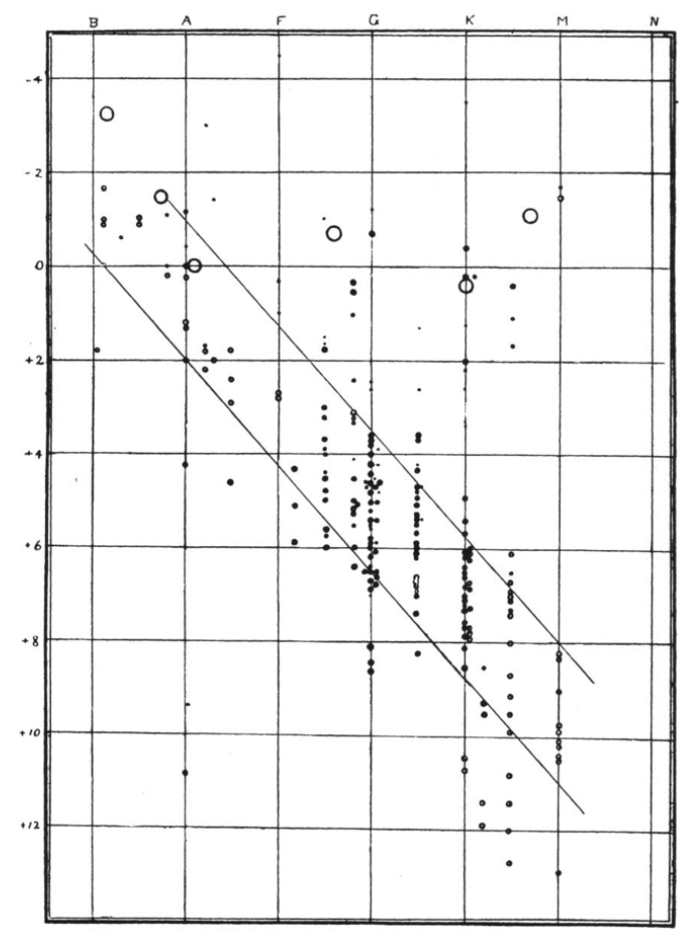

FIGURE 1. An early H-R Diagram taken from [26]. The only star in the bottom left corner is the WD 40 Eridani, although it was not known to be a WD at the time.

product of hydrogen fusion) begins to collapse while its outer layers swell in size, becoming a red giant star. Eventually the core becomes hot enough to begin fusing helium to heavier elements, including carbon and oxygen. While interesting in its own right, for our present purposes, we will skip over the details of this stage of a star's evolution.

Eventually the outer layers of the star are shed, as a planetary nebula, and the hot core is left exposed. This leftover core is a WD star. As I mentioned previously, it is composed mostly of carbon and oxygen, the result of the helium fusion stage. This is where we begin our investigation of the applications of these simple and versatile stars.

\subsection{White Dwarf Cooling}

Because there is no internal fusion occurring, a WD star shines because it is so hot, with its initial temperature often exceeding $100,000 \mathrm{~K}$. With no internal heat sources, over time the WD will begin to cool and, after some time, begin to crystallize. Eventually it will reach the temperature of its surroundings, fading completely out of our sight. The evolutionary path a star takes on the H-R Diagram from the MS to a cooling WD is indicated by the gray line in Figure 2 (taken from [33]). (The regions labeled as "DOV," "DBV," and "DAV" are the various instability strips a WD will pass through as it cools. I will discuss these more later.)

Mestel [18] was the first to describe and quantify how the rate of cooling of a WD relates to its luminosity, using standard cooling physics. Since then, it has been realized that there are 


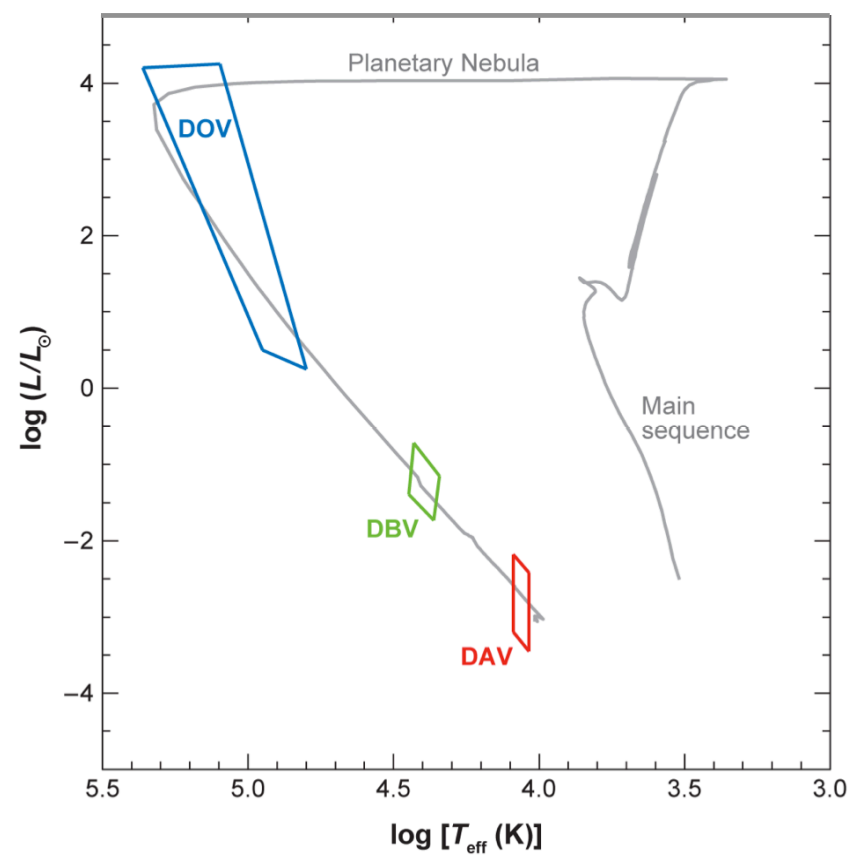

FIGURE 2. A schematic of the evolutionary track of a typical WD on the H-R Diagram, taken from [33]. The regions marked "DOV," "DBV," and "DAV" are the various instability strips a WD passes through in its evolution.

five physical effects during a WD's cooling that can provide a deviation from this classical theory. These effects, as described by [15], are as follows: (1) initial neutrino loss; (2) possible residual nuclear burning in the outer hydrogen layer; (3) gravitational contraction; (4) surface convection; and (5) crystallization.

For simplicity in our current discussion, I will classify WD cooling into three main stages: "hot," "warm," and "cool." While "hot" and "cool" are used commonly throughout the WD community, describing a WD as "warm" is somewhat unconventional; but I have chosen it here to clarify the discussion of those WDs that are neither classified as "hot" nor "cool," but rather have temperatures somewhere in between these two.

\section{Applications of White Dwarf Science}

Several of the properties of WDs also make them very useful as probes to address several astrophysically interesting problems. As the eventual fate of $98 \%$ of the stars in our Galaxy, they contain an archeological history of our own Milky Way. The conditions of high density and temperatures on a WD make them extreme physics laboratories, allowing us to test the behavior of material in an environment that cannot be replicated in the laboratory on Earth. When compared to their MS counterparts, WD stars are relatively simple and therefore easier to model and understand.

Some of the applications of WD science that I will discuss below include probing questions related to particle physics (specifically neutrinos); asteroseismology; searching for 
planets, dust and debris disks of remnant solar systems; understanding the origins of Type Ia supernovae; and measuring the ages of stellar populations, including star clusters (both open and globular clusters) and constituents of our Milky Way Galaxy. The study of WDs in clusters is especially interesting for a wide variety of topics; finding WDs in clusters allows us to fit WDs into the whole picture of stellar evolution and address many outstanding issues.

\subsection{Hot White Dwarfs}

The hottest WDs mark the transition between the planetary nebula phase and the WD phase. A study of the very hottest WDs is important for understanding this important transition in stellar evolution. When these WDs have cooled to roughly $18,000-28,000 \mathrm{~K}$ they will begin to pulsate (classified as DBVs). (The "DB" classification indicates the WD stars with observed helium in their atmospheres; the "V" classification indicates it is a variable WD.) Like all WD pulsators, they are non-radial pulsators. Unlike radial pulsating stars that change brightness because of actual changes in the size and effective temperature of the star, non-radial pulsators vary in brightness because of temperature fluctuations over their surface. Typically, they are multi-periodic and can show more complicated light curves than radial pulsators. This is illustrated in Figure 3, taken from [33], which shows the modeled surface brightness (on the right) along with the corresponding observed light output (i.e., photon flux, shown on the left) at various times for a given star.

A WD, like any hot object, initially cools very quickly. With time that cooling rate begins to slow. A WD's thermal energy is being carried away by photons, but also by other particles, including neutrinos and axions. In the early hot stages of a WD's cooling, the neutrino flux can actually be greater than the photon flux. As I mentioned above, it has been noted that the rate at which a WD actually cools is affected by the initial neutrino losses. For the hottest WDs in the DBV instability strip, the WD is still hot enough that its cooling is still dominated by neutrino losses. This effect is illustrated in Figure 4 (taken from [14]). Observations of the rate of change of the period of DBV stars allow us to measure their cooling rate. By comparing the expected cooling rate from photons alone to the observed cooling rate, we are able to probe the properties of these exotic particles that are being emitted from the WD star. (See [1] for more details on this application of WD studies.)

\subsection{Warm White Dwarfs}

Some of the earliest uses of WDs to probe various astrophysical questions came from studying the warm WDs. For example, the largest class of pulsating WD stars actually belong to the DAV class. (The "DA" classification indicates the WD stars with observed hydrogen in their atmospheres; the "V" classification indicates it is a variable WD.) These are WDs with hydrogen-rich atmospheres that are roughly 12,000 K. Also known as the ZZ Ceti stars, this class of WD pulsators was first discovered by Landolt (1968), and there are about 143 known members today ([9]; [2]). (Compare this to the DBVs, with roughly 18 members, [22]; [23]). 


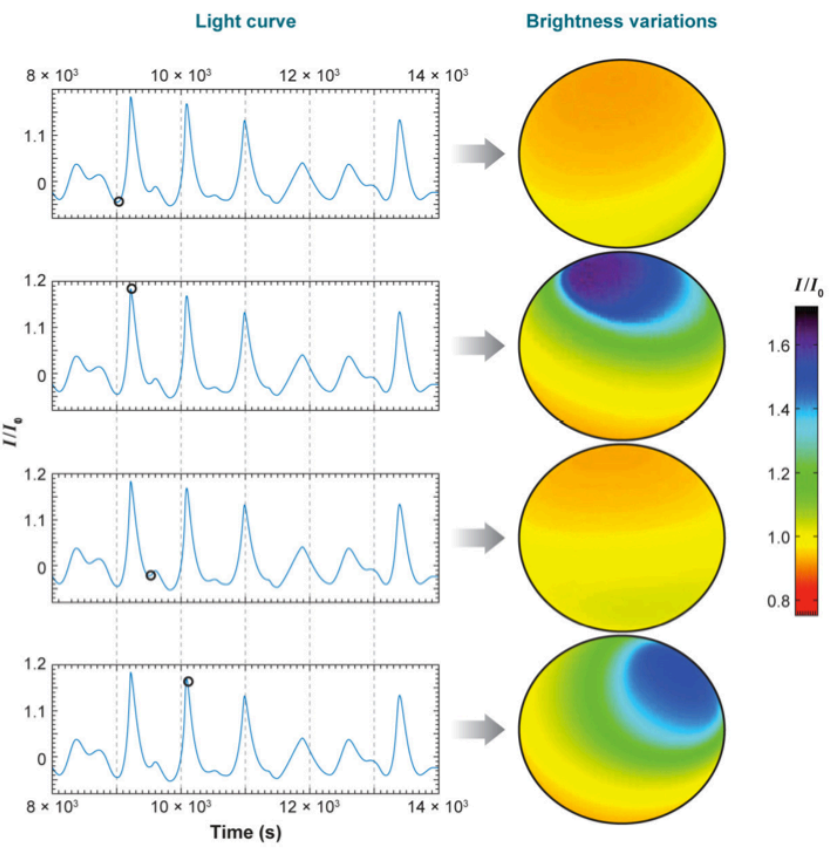

FIGURE 3. Surface brightness changes for a non-radial pulsating WD star. The left side shows the observed brightness (photon flux) corresponding to surface brightness changes modeled on the right. (Taken from [33].)

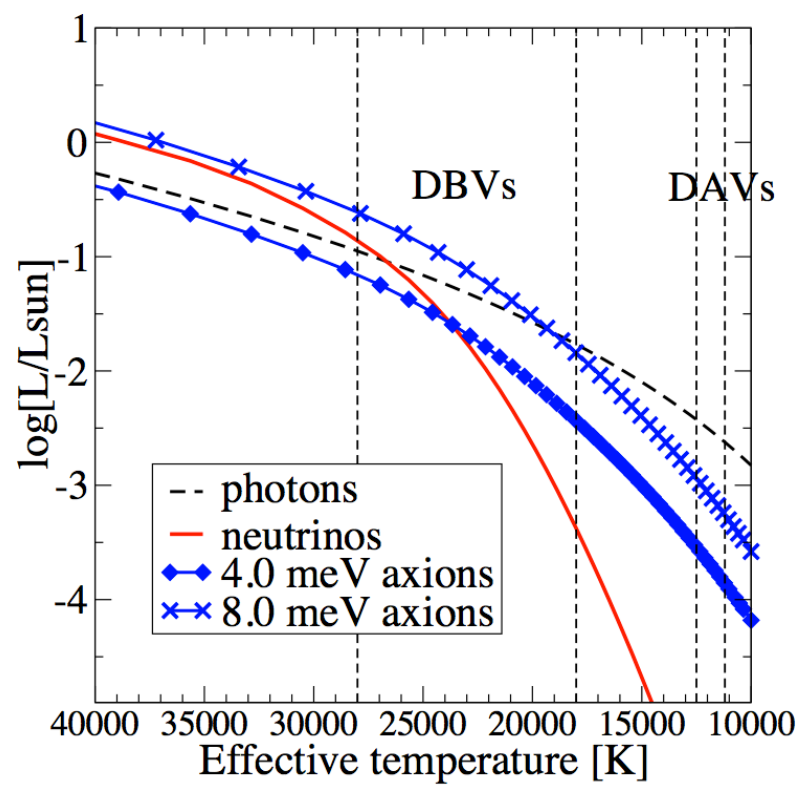

FIGURE 4. Expected flux from photons, neutrinos, and axions as a WD initially cools. Note that initially the flux from the neutrinos and $8.0 \mathrm{meV}$ axions are greater than the photon flux. This trend continues into the DBV instability strip. Measuring the cooling rate for hot DBVs and comparing to the expected cooling rate from photons alone can give insight to the nature and amount of other particles carrying away thermal energy from the WD. (Plot taken from6[14].) 
DAV stars have been used in many applications. Astereoseismology of these stars can be used to probe their interiors. For example, this allows for a more thorough study of crystallization as the WD cools (e.g., [20]). Studies of the pulsations can also be used to measure the mass of the star and the mass of its layers ([33]), as well as to study the processes of convection ([19]).

More recently DAVs have been used to search for planets. Finding planets around WD stars is an exciting prospect because these WDs could give insight into the eventual fate of our own Solar System. (Particularly because our Sun will one day become a WD star.) Because the pulsation periods of some DAV stars are extremely stable, any periodic change in the pulse arrival time may indicate the wobble of a star due to an unseen companion as the two stars orbit their common center of mass. Such work has been started by [21] and built upon by [8]. Figure 5 , taken from [8] shows a plot of observed minus calculated $(\mathrm{O}-\mathrm{C})$ values of pulse arrival times for the star GD66. The blue sinusoid corresponds to the effect on a DAV caused by a $2.7 \mathrm{M}_{\mathrm{J}} \sin i$ planet at $4.9 \mathrm{AU}$ with a 14.3 year orbit.

Related to the search for planets around other stars is the work looking for debris or dust disks around WD stars. For example, infrared observations of some WD stars indicate excess flux that is indicative of a debris disk around the star (see, e.g., [13]). Also, [27] have studied the presence of calcium across the surface of G29-38 using time-series spectroscopy (see Figure 6 , taken from this study). Because the metals would settle on short time scales, the continual presence of calcium in the spectrum indicates that it is being replenished, likely from the debris disk that surrounds it.

\subsection{Binary White Dwarfs}

WD stars are candidate progenitors for Type Ia supernovae (SNIa). One hypothesis is that when a WD is in a binary system with a less evolved star, and that star expands to fill its Roche Lobe, the WD begins accreting material onto its surface. As it gains more and more mass it soon reaches the Chandrasekhar limit. When this happens, the WD explodes. Because the Chandrasekhar limit is universally the same, all SNIa have the same brightness and can be used as standard candles to measure distances to the furthest galaxies. SNIa were used to discover the acceleration of the Universe and continue to be an important cosmological tool.

However, saying that every SNIa has exactly the same luminosity is oversimplifying the problem. Effects such as crystallization, phase separation, dynamical and pulsational modes of energy transport, the dependence of mass loss on metallicity, and other aspects of WD physics must be understood. These factors may affect our interpretation of variations in the brightness of SNIa with redshift, and subsequently affect our interpretation of cosmological observations and implications.

\subsection{Cool White Dwarfs}

Early observations of the WD luminosity functions (LFs) of the Galactic disk (e.g., [17]) showed a steep drop off at the faint end. Theoretical calculations of the WDLF by [34] showed that the observed turndown of the WDLF was indeed a measure of the age of the Galactic disk. I display the early WDLF fit with theoretical models in the left panel of Figure 7, taken from 


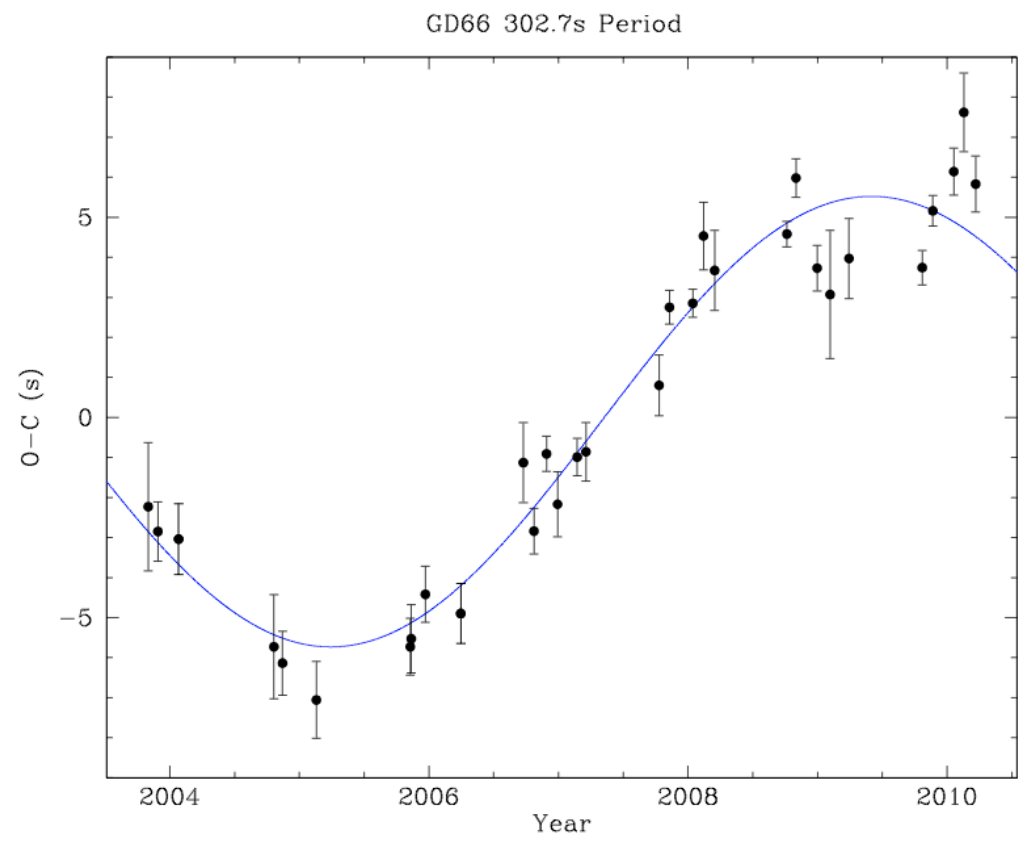

FigURE 5. Taken from [8], the $\mathrm{O}-\mathrm{C}$ diagram for the $302.7 \mathrm{~s}$ period mode of GD 66 . The observed changes in pulse arrival time are too great to be explained by the cooling of the star and are likely indicative of a companion planet.

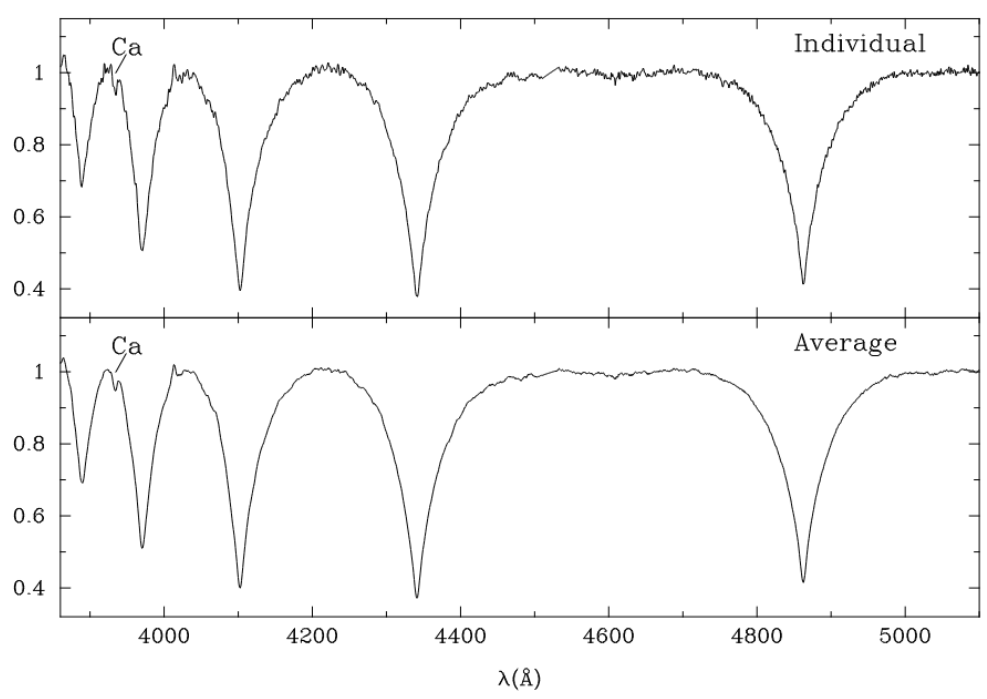

FIGURE 6. Spectra of GD29-38, taken from [27]. Note the presence of a calcium feature. Because metals would settle out of the atmosphere on extremely short timescales, the continual presence of calcium indicates that it must be replenished by some external source (e.g., dust disk, small planetary bodies, etc.). 

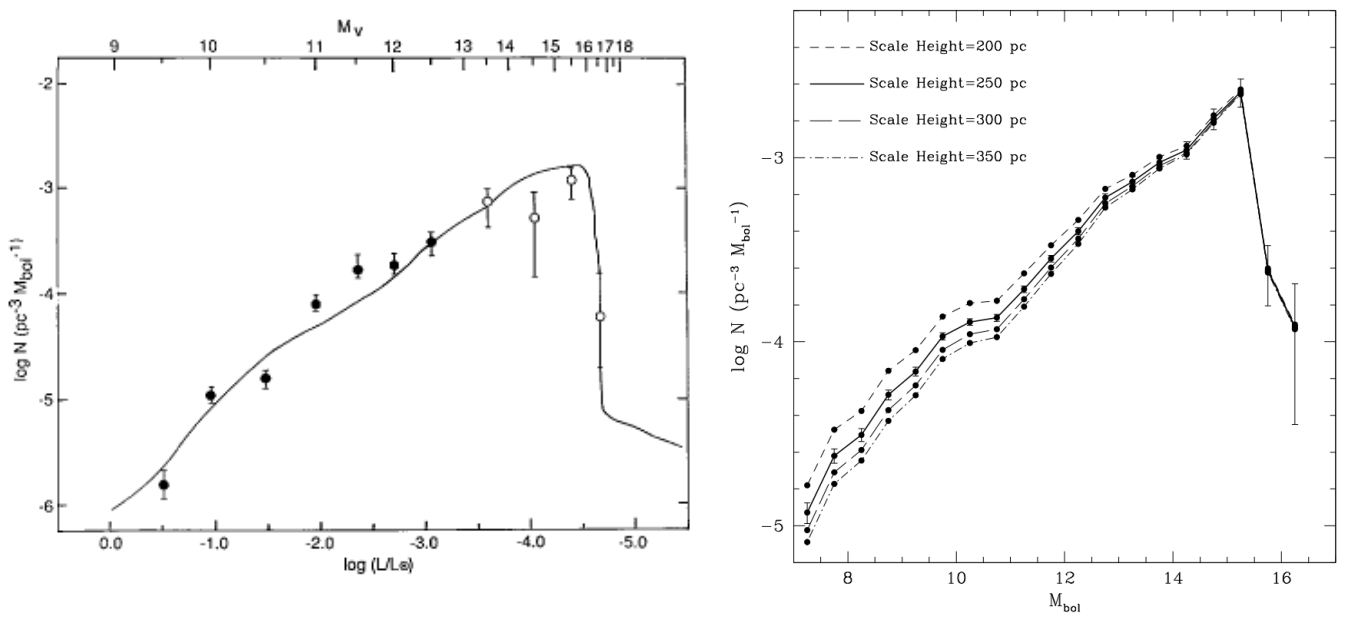

FIGURE 7. WDLF for the Galactic disk. It was first theoretically calculated by [34], left panel) that the WDLF could be used to determine the age of the disk. Over time, more WDs have been added to the WDLF and calculations and models continue to progress (left panel, taken from [7]).

[34]. The reason for this turndown is that the finite age of the disk does not allow the WD stars to cool beyond a certain luminosity. Because the luminosity of a WD star is related to its cooling time ([18]) and hence WD age, the turndown luminosity of a population's WDLF was therefore related to the age of the population. This work also demonstrated the feasibility of using the WDLF to constrain aspects of the star formation history of the Galactic disk.

In the roughly two decades that have passed between these initial studies, sample sizes have increased (e.g., [7]) to allow for us to disentangle effects from such things as WD mass distributions (e.g., [4]), as well as to extend the use of WDs as chronometers to various scale heights of the Galaxy. Work has been done to further improve both the observations and theoretical calculations of the WDLF. An example of this is the recent sample of [7], reproduced in the right panel of Figure 7.

\subsection{White Dwarfs in Star Clusters}

Star clusters are Nature's stellar laboratory. By removing uncertainties in the star-to-star differences in distance, age, reddening, and metallicity that plague field-star work, we are able to study the evolution of stars in an essentially pure environment. Observations of WDs in open and globular clusters have reached an exciting development in the last decade or two, thanks to the advancement of large ground-based and space telescopes. In this section I will briefly discuss some of the exciting questions that can now more fully be addressed by studying WDs in the controlled environment of a star cluster. 


\subsubsection{Maximum Mass for a White Dwarf Progenitor}

Not all stars will become WDs. The stars that are massive enough will reach the endpoint of their evolution as either a neutron star or a black hole. But the key question is: how massive is "massive enough" to be considered high mass? Which stars will become WDs and which stars won't? Star clusters can be used to answer this question, by connecting the mass of stars at a cluster's main sequence turn off (MSTO) to the presence (or lack) of WDs. The current best estimate for this value is $8 \mathrm{M}_{\odot}[32]$.

\subsubsection{Initial-Final Mass Relation}

The mass distribution for WD stars in the field shows a fairly sharp peak at approximately $0.6 \mathrm{M}_{\odot}$. However, the mass of the WD progenitors shows a much broader range. Our understanding of the processes involved in mass loss from when a star leaves the MS to when in becomes a WD is poor, at best. There is much we do not understand. Star clusters can aid us in this process as well. Studying the initial-final mass relation (IFMR) - that is, the relationship between a star's initial MS mass and its final WD mass - can only be done reliably in star clusters. Studying the IFMR in many clusters can shed light on the processes of mass loss, as well as give indications of metallicity dependence on mass loss.

\subsubsection{Observations of White Dwarfs in Globular Clusters}

An important and exciting advancement in the study of WDs in clusters is the observation of the terminus of the WD cooling track in three globular clusters. These clusters are M4, NGC 6397, and 47 Tuc. These observations provide information about cluster ages, crystallization (see Section 3.5.4), and collisionally-induced absorption opacities in these extremely old WDs. These studies are being aided by the superb datasets made available from the Hubble Space Telescope, especially in proper-motion cleaned color-magnitude diagrams. In Figure 8, I display the color-magnitude diagram for NGC 6397 with pre- and post-proper motion cleaning. (These figures were taken from [24].)

\subsubsection{Crystallization of White Dwarfs}

Observations of the terminus of WDs in globular clusters have created a rich dataset for a variety of studies. One of these applications is the ability to study the crystallization of the oldest cluster WDs as they cool. Winget et al. [35] fit models to observations of the WDs in NGC 6397 to demonstrate the effects of crystallization on the cooling of the WDs. (See Figure 9 , taken from their paper.)

\subsubsection{Star Cluster Ages}

As discussed in Section 3.4, WDs can be used to determine the age of a stellar population. The same methods discussed there of finding the coolest WDs in the population can be applied to star clusters. The earliest attempts to do this were done by [3] and [30]. In Figure 10 I show a plot of the current agreement between open clusters from [11], an updated version of the same plot from [29].

An important motivation of these studies is to calibrate ages determined from the MSTO and those measured from the WDs. These two age methods are used to measure the ages of 

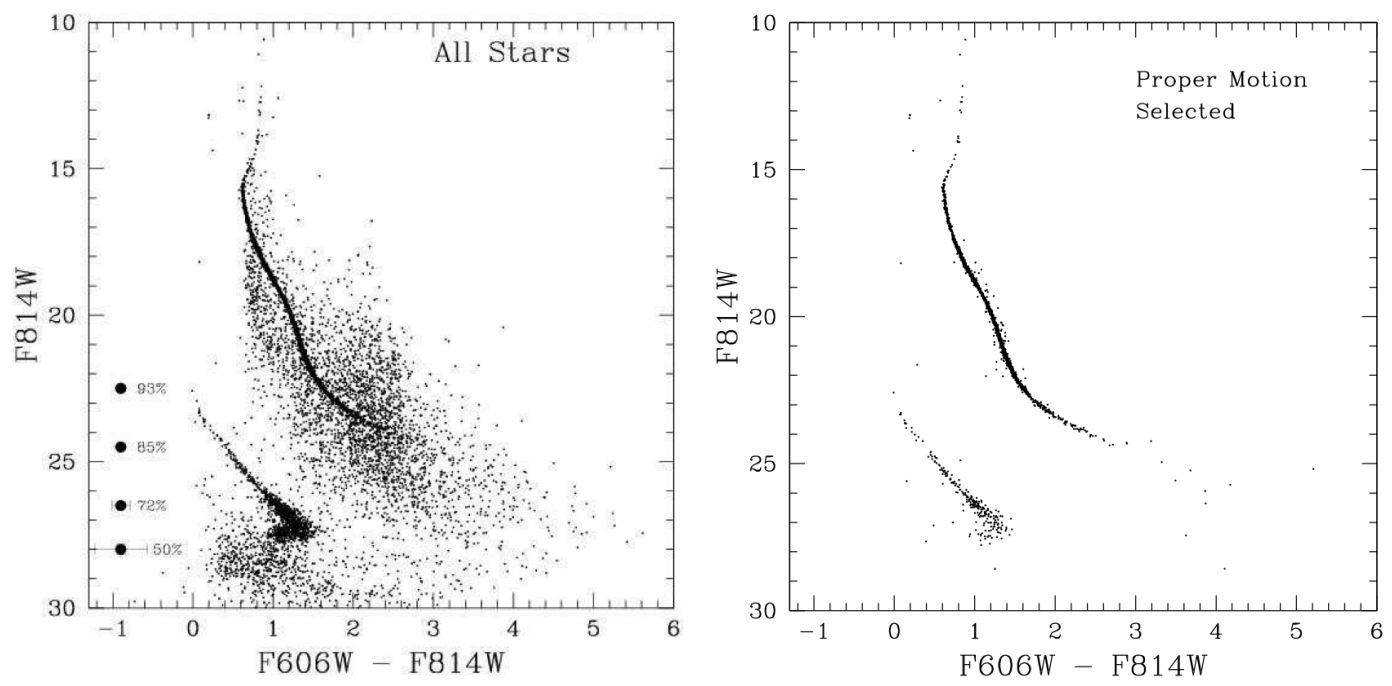

FIGURE 8. Color-magnitude diagram for NGC 6397 [24]. Data are taken with the Hubble Space Telescope; the panel on the left is the entire field, while the right panel is the proper motion cleaned, showing only those stars that are most likely to be cluster members

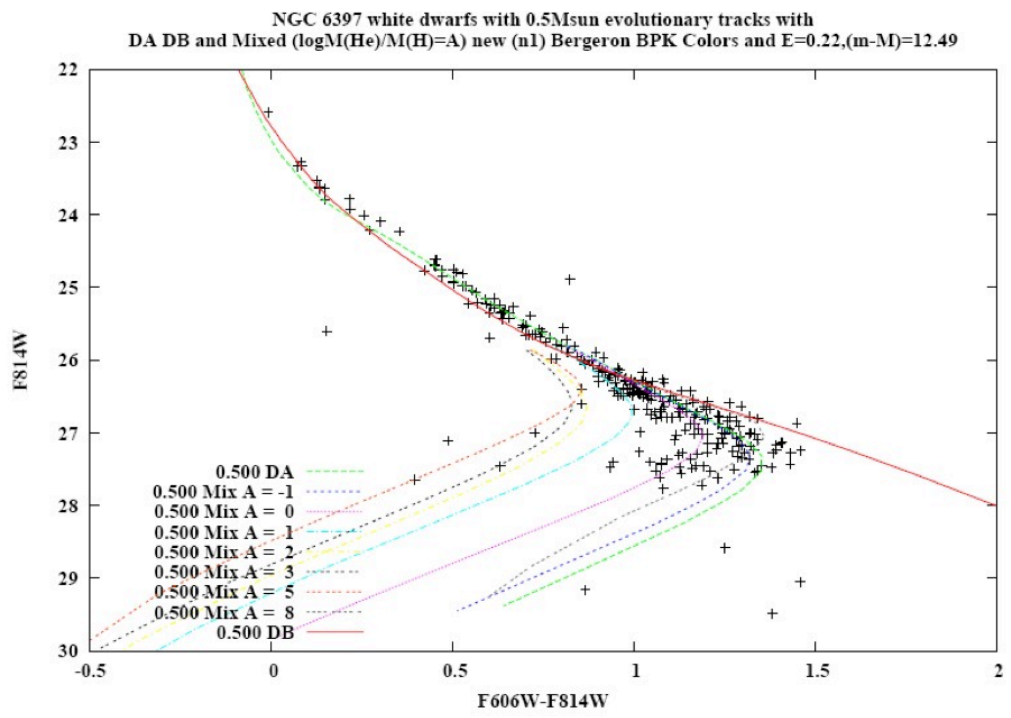

FIGURE 9. The WD cooling sequence of NGC 6397 with various models to investigate the effects of crystallization on the observed WDs. (Taken from [35].)

different parts of the Galaxy: the best age of the halo from the MSTO of globular clusters and the best age of the disk from the WDLF (Section 3.4). Uncertainty exist in the models used for both methods. MSTO model uncertainties includes nuclear reaction rates, uncertainties in the population's metallicity, the equation of state, as well as second-order effects including diffusion, rotation, and turbulence. Uncertainties in WD ages can arise from the mass 


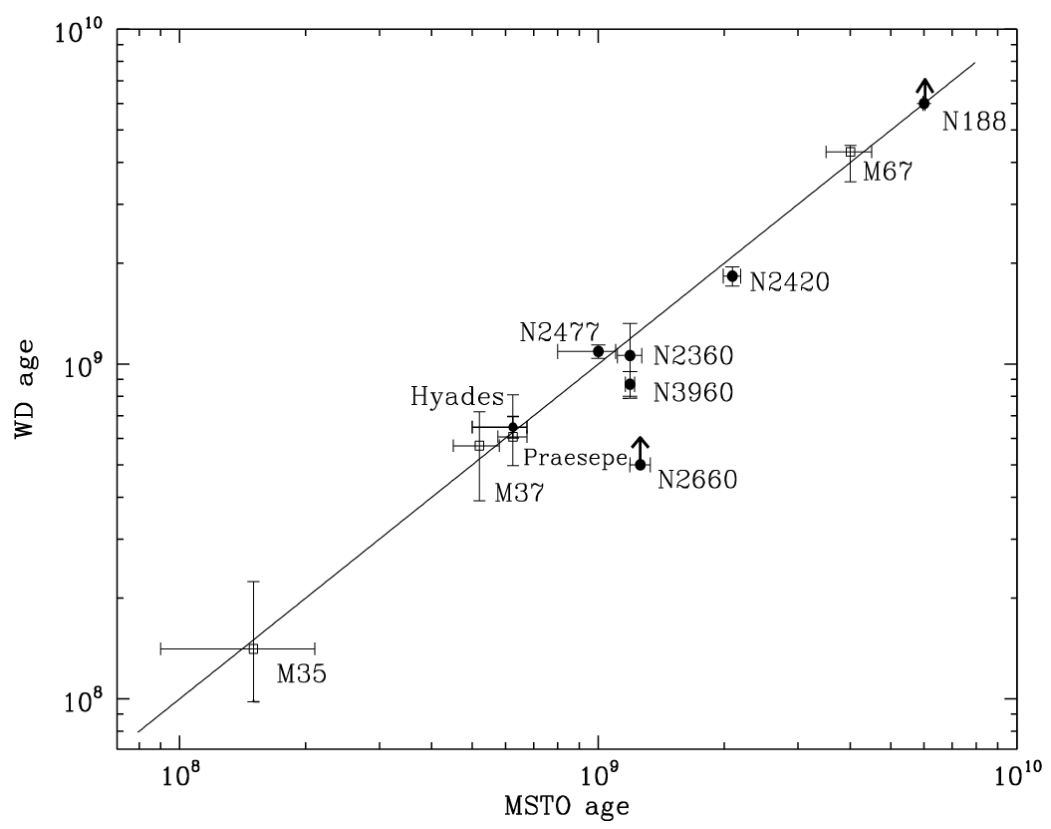

FIGURE 10. The current agreement of WD age vs. MSTO age for open clusters, taken from [11], an updated version of the original plot from [29].

distribution, the onset of core crystallization, molecular hydrogen opacities, and unknown DA/DB ratios. Because each model relies on different physics with different limitations, measuring the ages of clusters with each method allows us to more directly determine and isolate problems in the models, based on any disagreement that may arise between the ages calculated for a single population.

Over time our ability to obtain data of higher and higher precision has increased. However, in the coming years, the greatest improvements in age determinations of clusters will likely come in improvements in modeling. One such improvement is being developed by von Hippel and collaborators, using an algorithm that utilizes Bayesian statistics to objectively and simultaneously fit cluster-wide parameters such as age, metallicity, distance, and reddening to input models. (For more information, see [31], [12], [28], and [5].) Part of the power of the technique lies in being able to compare the posterior distribution for each of these parameters among a variety of model sets.

For example, in Figure 11 (taken from [11]), comparisons are made of the best age, metallicity, distance, and reddening determined from the best fit of three different MS model sets for the WD age of the open cluster NGC 2477. (In this case the different MS models were: [6]; [36]; and [10]. Other model ingredients were unchanged, but can - and will - eventually be varied for comparison. See [11] for further discussion on the exact model ingredients used.) The statistics that can be calculated from this method can now include internal errors (error within a particular model, i.e., the width of the distributions) and the external errors (errors 

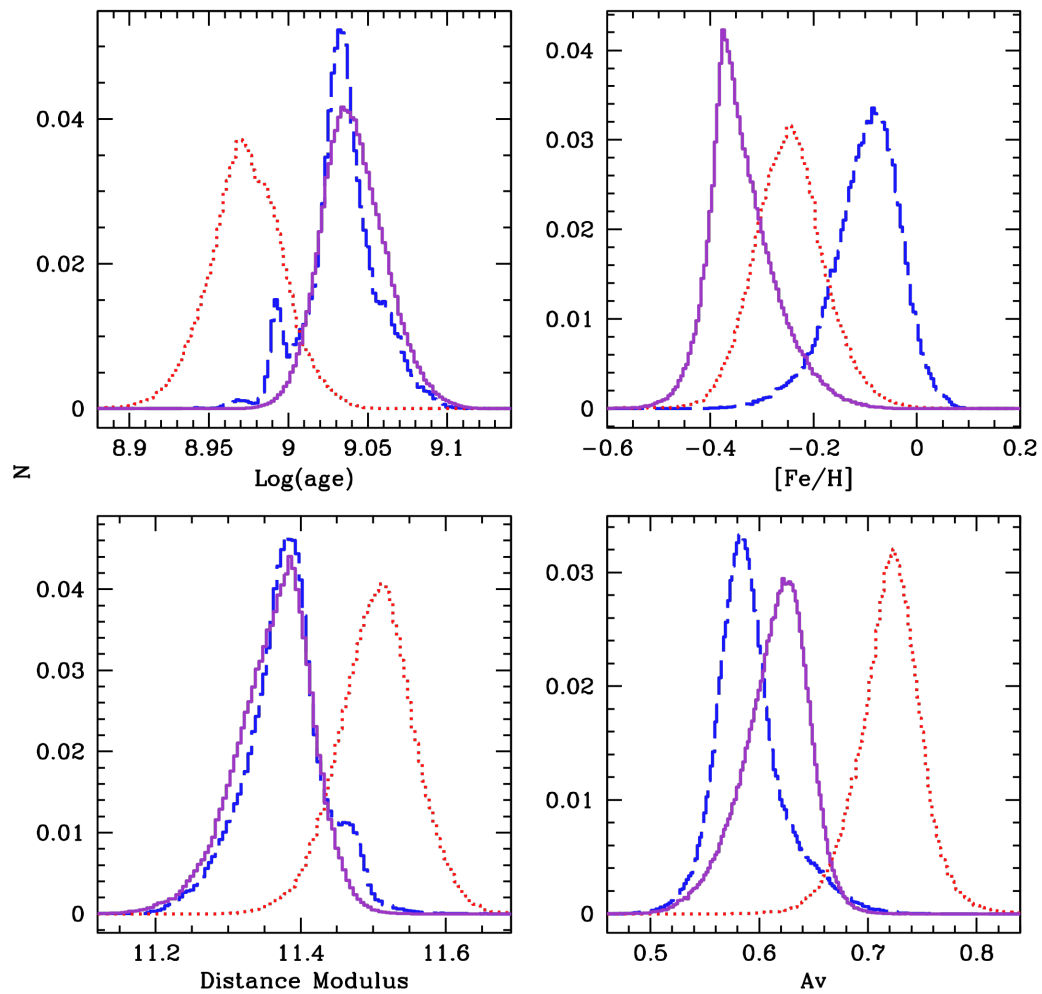

FIGURE 11. Posterior distributions for cluster-wide parameters of NGC 2477 (WD age) for three different MS models. The dashed lines are using the models of [6]; the dotted lines are using [36]; the solid lines are using [10]. The power of the Bayesian technique comes from being able to compare entire distributions, rather a single number.

among the models, i.e., taking into account the differences in the different distributions).

\section{Summary}

WDs, the endpoint of the evolution of $98 \%$ of stars, are simple stars, but have far-reaching applications to many fields of astrophysics. From hot WDs to cool WDs, some applications include particle and extreme physics, asteroseismology, the study of planets, understanding the expansion of the Universe (through Type Ia supernovae), measuring the ages of stars, and understanding important aspects of stellar evolution with the study of WDs in star clusters. In this paper I have reviewed a few of the applications of these interesting stars.

With the improvement of datasets and modeling techniques, we will be able to continue to push the boundaries of WD physics. There are still many puzzles in WD physics to study and explore. Doing so will improve the usability of these interesting objects and increase our understanding of many aspects of the Universe around us. 


\section{References}

[1] Bischoff-Kim, A. et al., 2008, ApJ, 675, 1505

[2] Castanheira, B.G., et al. 2007, $A \& A, 462,989$

[3] Claver, C.F. 1995, PhD Thesis, The University of Texas at Austin

[4] DeGennaro, S. et al. 2007, $A J, 135,1$

[5] DeGennaro, S. et al. 2009, ApJ, 696, 12

[6] Dotter, A. et al. 2008, ApJS, 178, 89

[7] Harris, H.C., et al. 2006, $A J, 131,571$

[8] Heremes, J.J., et al. 2010, Proceedings of the $17^{\text {th }}$ European White Dwarf Workshop, AIP Conference Proceedings, 1273, 446

[9] Gianninas, A., et al. 2006, $A J, 132,831$

[10] Girardi, L. et al. 2000, $A \& A S, 141,371$

[11] Jeffery, E.J. 2009, PhD Thesis, The University of Texas at Austin

[12] Jeffery, E.J. et al. 2006, $A p J, 658,391$

[13] Jura, M. et al., 2009, $A J, 137,3191$

[14] Kim, A. 2007, PhD Thesis, The University of Texas at Austin

[15] Lamb \& Van Horn, 1975, ApJ, 200, 306

[16] Landolt, A.U. 1968, ApJ, 200, 306

[17] Liebert, J. et al. 1988, ApJ, 332, 891

[18] Mestel, L. 1952, MNRAS, 112, 583

[19] Montgomery, M.H., et al. 2005, ApJ, 633, 1142

[20] Montgomery, M.H. \& Winget, D.E. 1999, ApJ, 526, 976

[21] Mullally, F. et al. 2008, $A p J, 676,966$

[22] Nitta, A., et al. 2007, Commun. Asteroseismol., 150, 249

[23] Nitta, A., et al. 2009, ApJ, 690, 560

[24] Richer, H.B. et al. 2008, $A J, 135,2141$

[25] Russell, H.N. 1913, The Observatory, 36, 324

[26] Russell, H.N. 1914, Nature, 93, 52

[27] Thompson, S.E. et al. 2010, ApJ, 714, 296

[28] van Dyk, D.A., et al. 2009, The Annals of Applied Statistics, 3, 117

[29] von Hippel, T. 2005, ApJ, 622, 565 
[30] von Hippel, T., et al. 1995, MNRAS, 273, L39

[31] von Hippel, T. et al. 2006, ApJ, 645, 1436

[32] Weidemann, V. \& Koester, D. 1983, $A \& A, 121,77$

[33] Winget, D.E. \& Kepler, S.O. 2008, ARA\&A, 46, 157

[34] Winget D.E., et al. 1987, ApJ, 315, L77

[35] Winget, D.E., et al. 2009, ApJ, 693, L6

[36] Yi, S., et al. 2001, ApJS, 136, 417 